\title{
IL FONDAMENTO ETICO DEI DIRITTI UMANI
}

I grandi problemi che si pongono agli esseri umani hanno ormai una dimensione planetaria, poiché lo sviluppo delle tecniche mass-mediali favorisce una crescente interazione tra le persone, le società e le culture in un mondo che si va sempre più globalizzando, in quello che Mc. Luhan ha chiamato "villaggio globale". Ogni cultura è trascendenza e superamento. Se si arresta, come ammonisce Mounier, diventa incultura, accademismo e, se confonde l'universalità con la totalità statica, si cristallizza nel sistema.

Emerge cosi la consapevolezza di una solidarietà globale, che trova il suo fondamento ultimo nell'unità del genere umano. Questa si traduce in una responsabilità morale a livello planetario e comporta la ricerca di valori etici condivisi. La crescente interdipendenza tra i popoli, mentre richiede il rifiuto del terrorismo e della violenza come via principale per ricostruire le condizioni essenziali di giustizia e libertà, esige soprattutto una forte solidarietà morale, culturale, economica e un'organizzazione politica della società internazionale che possa garantire i diritti di tutti i popoli. La soluzione al male del sottosviluppo e alle situazioni drammatiche in cui vivono e muoiono milioni di persone è di natura fondamentalmente etica, e ad essa devono corrispondere scelte economiche e politiche coerenti. Il primo decisivo contributo per uno sviluppo veramente degno dell'uomo è rappresentato dal sostegno a programmi di educazione culturale, sottesi da un'etica della responsabilità.

Non mancano i tentativi contemporanei per delineare un'etica universale. Dopo il secondo conflitto mondiale la comunità delle nazioni ha definito nella Dichiarazione Universale dei diritti dell'uomo del 1948 alcuni diritti inalienabili della persona umana; essi non sono concessi semplicemente dal legislatore, ma sono dichiarati poiché la loro esistenza è anteriore al legislatore. Nella Dichiarazione, adottata dalla assemblea generale delle Nazioni Unite, figura un paragrafo significativo: «... è essenziale che i diritti dell'uomo siano protetti da un regime di diritto affinchè l'uomo non sia costretto, come estremo ricorso, alla rivolta contro la tirannia e l'oppressione»' ${ }^{\text {, }}$ in tale redazione riappare la vexata quaestio della fondatezza giuridica di uno ius 
resistentiae, che ripropone il classico quesito: in quali modi è lecito difendere la libertà? Un interrogativo di fondo, la cui portata supera l'ambito giuridico per investire il piano etico, quello della coscienza chiamata a operare un discernimento sempre esigente, valutando responsabilmente i mezzi per appoggiare la giustizia e opporsi all'ingiustizia. Di questo si tratta, in definitiva, quando si parla di rispetto o di violazione dei diritti dell'uomo. La Dichiarazione, come ricorda Giovanni Paolo II, rimane una delle "espressioni più alte della coscienza umana nel nostro tempo", ma i risultati non sempre sono stati forieri di buone speranze, giacchè emerge sempre più una tendenza a reinterpretare i diritti dell'uomo separandoli dalla dimensione etica e razionale, che costituisce il loro fondamento e il loro fine per un puro legalismo utilitarista ${ }^{2}$.

Per spiegare il fondamento etico dei diritti dell'uomo alcuni hanno cercato di elaborare un" "etica mondiale" nell'ambito di un dialogo tra le culture e le religioni. «L'etica mondiale indica l'insieme dei valori obbligatori fondamentali che da secoli formano il tesoro dell'esperienza umana, la quale è rinvenibile in tutte le grandi tradizioni religiose e filosofiche (...). Tale progetto, degno di interesse, è espressione del bisogno attuale di un'etica che abbia validità universale e globale ${ }^{3}$.

Quattro sono i principi contenuti nella Dichiarazione per un'etica planetaria, formulata da alcuni esponenti del parlamento delle religioni del mondo nel 1993, la quale afferma che esiste tra le religioni un consenso minimo che riguarda valori obbliganti, norme irrevocabili e tendenze morali essenziali:

1) nessun nuovo ordine del mondo senza un'etica mondiale;

2) ogni persona umana sia trattata umanamente;

2 Cfr. Benedetto XVI, Discorso del 18 Aprile 2008 davanti all'Assemblea generale dell'On u, in AAS 100 (2008) 335. II Papa cosi si esprime: «ll merito della Dichiarazione Universale è stato di aprire a culture, a espressioni giuridiche e modelli istituzionali diversi la possibilità di convergere attorno a un nodo fondamentale di valori e quindi di diritti: ma è uno sforzo che oggi dev'essere ancora più sostenuto di fronte a istanze che cercano di reinterpretare i fondamenti della Dichiarazione e di comprendere l'unità interna per favorire il passaggio dalla protezione della dignità umana all'appagamento di semplici interessi, spesso particolari (...). Sovente constatiamo nei fatti un predominio della legalità sulla giustizia, quando si manifesta un'attenzione alla rivendicazione dei diritti che giunge sino a farli apparire come il risultato esclusivo di disposizioni legislative o di decisioni normative prese dalle diverse istanze della autorità in carica. I diritti, quando sono presentati sotto una forma di pura legalità, rischiano di diventare proposizioni di debole portata, separati dalla dimensione etica e razionale, che costituisce il loro fondamento e il loro fine. La Dichiarazione universale ha infatti riaffermato con forza la convinzione che il rispetto dei diritti dell'uomo è radicata prima di tutto in una giustizia immutabile sulla quale è pure fondata la forza coercitiva delle proclamazioni internazionali. $\grave{E}$ un aspetto che spesso è trascurato, quando si pretende di privare i diritti della loro vera funzione in nome di una stretta prospettiva utilitarista».

3 Cfr. lo studio Alla ricerca di un'etica universale: Nuovo sguardo sulla legge naturale, n. 6 in La Civiltà cattolica, 2009 II, p. 344. Questo documento elaborato dalla Commissione Teologica Internazionale (d'ora in poi CTI) in occasione delle sessioni plenarie della stessa CTI, tenutesi a Roma, nell'ottobre 2006-2007 e nel dicembre 2008, è stato poi approvato all'unanimità nella sessione 1-6 dicembre 2008 ed è stato poi sottoposto al suo presidente, il cardinale William J. Levada, che ha dato la sua approvazione per la pubblicazione. 
3) la Dichiarazione enuncia quattro direttive morali: non violenza, e rispetto della vita; solidarietà; tolleranza e verità; uguaglianza dell'uomo e della donna;

4) riguardo ai problemi dell'umanità è necessario un cambiamento di mentalità, affinchè ciascuno possa prendere coscienza della propria responsabilità. ${ }^{4}$

Già da molti anni verso la fine del XX e l'inizio di questo terzo millennio la questione dei fondamenti etici del diritto e della stessa politica è stata messa da parte in molti settori della cultura contemporanea con il pretesto che ogni pretesa di una verità oggettiva e universale sarebbe fonte di intolleranza e di violenza, e che soltanto il relativismo potrebbe salvaguardare il pluralismo dei valori e la democrazia.

Si va da più parti sostenendo il positivismo giuridico che rifiuta di riferirsi a qualcosa di assoluto, fondante, a un criterio oggettivo, ontologico, di ciò che è giusto. In questa prospettiva, l'ultimo orizzonte del diritto e della norma morale è la legge in vigore, che è considerata giusta per definizione, poiché è espressione della volontà del legislatore. È superfluo aggiungere che una simile posizione apre la via all'arbitrio del potere, alla dittatura della maggioranza aritmetica e alla manipolazione ideologica a scapito del valore del bene comune. Giovanni Paolo II, nell'enciclica Fides et ratio, auspica come «necessaria una filosofia di portata autenticamente metafisica, capace cioè di trascendere i dati empirici per giungere nella ricerca della verità, a qualcosa di assoluto, di ultimo, di fondante. È un'esigenza, questa, implicita sia nella conoscenza a carattere sapienziale che in quella a carattere analitico; in particolare è un'esigenza propria della conoscenza del bene morale, il cui fondamento è il bene sommo, Dio stesso» ${ }^{5}$.

Il razionalismo moderno pone la ragione come criterio unico del vero, del bene, del giusto e pretende di avere certezze assolute senza presupposti escludendo l'idea $\mathrm{d}$ Dio che si è manifestato nella storia con Cristo, in favore di una morale da seguire "etsi Deus non daretur" ponendo Dio tra parentesi. È nota al riguardo la formulazione kantiana dell'autonomia della morale che esclude Dio nella riflessione filoso-

Nell'incontro di giugno 1993, 34 governi arabi ed asiatici pubblicarono la Dichiarazione di Bangkok, in cui venne affermato che la nozione di diritti umani è relativa alla diversità culturale, religiosa e storica delle nazioni e che le potenze occidentali non dovrebbero usare i diritti umani come strumento di pressione politica (Universality of Righth is difended by U.S.; protesto of Dalai Lama Mars Vienna Talks, "The Washington Post", June 15, 1993, Sec. 1, p. 15). II documento finale, nonostante le diverse idee, afferma una posizione essenzialmente universalista: «l diritti umani e le libertà fondamentali sono per nascita diritti di tutti gli esseri umani (...). Tutti i diritti umani sono universali, indivisibili, interdipendenti e interrapportati» (Word Conference on Human Rights: The Vienna Declaration and Programma of Action, June 1993, New York United Nations Department of Pubblic Information, 1993, pp. 28-29). Tale documento sottolinea con forza i diritti delle donne, dei bambini, delle minoranze etniche e dei soggetti in difficoltà, e specifica che "il diritto di sviluppo" non può mai giustificare l'inflazione dei diritti umani perché «la persona umana è il soggetto centrale dello sviluppo» (ibidem, p. 31). Sulla centralità della persona e il fondamento etico dei diritti umani cfr. M. Indellicato, La centralità della persona nel pensiero di Jacques Maritain, Lecce 2009 
fica per non ledere la libertà dell'uomo ed aderire autonomamente al bene morale $\mathrm{e}$ alla moralità stessa. A questo punto vien spontaneo chiedersi: chi non ammette Dio e non riconosce che l'uomo è imago Dei su che cosa fonda la morale, i diritti umani e la dignità della stessa persona?

Se è vero che nel nostro tempo è maturata una coscienza sempre più viva circa la dignità della persona umana, del suo valore unico e irripetibile, e quindi del rispetto e dell'inviolabilità dei suoi diritti, è anche vero che la visione dell'uomo come persona è nata e si è affermata col cristianesimo, che ha posto la persona al centro della visione cristiana dell'uomo, immagine di Dio. Ovunque l'uomo scopre la presenza di un richiamo all'assoluto e al trascendente, lì gli si apre «uno spiraglio verso la dimensione metafisica del reale: nella verità, nella bellezza, nei valori morali, nella persona altrui, nell'essere stesso in Dio» ${ }^{6}$. Perciò, ammonisce ancora Giovanni Paolo II, la grande sfida che ci attende è quella «di saper compiere il passaggio, tanto necessario, quanto urgente, dal fenomeno al fondamento. Non è possibile fermarsi alla sola esperienza; anche quando questa esprime e rende manifesta l'interiorità dell'uomo e la sua spiritualità, è necessario che la riflessione speculativa raggiunga la sostanza spirituale e il fondamento che la sorregge»?

Ciò è tanto più necessario oggi quanto più assistiamo alla "crisi del senso", crisi cosi profonda che non pochi si chiedono «se abbia ancora senso porsi una domanda sul senso» e così si vive in «un orizzonte di totale assenza di senso» ${ }^{8}$, e ciò deriva proprio dal fatto che si rinunzia a «porre domande radicali sul senso e sul fondamento della vita umana, personale e sociale $\iota^{9}$. La crisi del senso, come crisi della stessa domanda di senso, la crisi del fondamento e la crisi di un pensiero metafisico, come capacità di accesso al fondamento, investono anche l'esperienza religiosa contemporanea. Il problema più grave con cui si deve confrontare oggi il cristianesimo in Europa è il nihilismo, mentre ieri era l'ateismo. "Con la morte di Dio", tanto osannata da Nietzsche, è arrivata la morte dell'uomo.Ciò che è in gioco, oggi, all'inizio del terzo millennio è la questione di senso. Il nihilismo è la crisi della speranza, del senso delle cose, della propria vita, dell'esistenza stessa; e questo perché non è riconosciuto il fondamento etico dei diritti umani, del nostro essere, del nostro vivere e convivere, e perché sono in crisi le ragioni ultime che orientano la vita e permettono che questa possa essere vissuta come un progetto. A tal proposito Bobbio, che definisce questo problema del fondamento o della giustificazione teorica "l'illusione del fondamento assoluto", afferma che non può essere posto in quanto tale e che il vero banco di prova dei diritti dell'uomo oggi non è tanto "quello di giustificarli, quanto quello di proteggerli" e cioè un problema non filosofico ma politico e l'unico

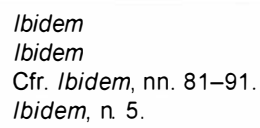


banco di prova della sua validità è «il consenso generale dell'umanità»» ${ }^{10}$. Poi aggiunge: «il fondamento assoluto non è soltanto una illusione; qualche volta è anche un pretesto per difendere posizioni reazionarie». ${ }^{11}$

Certamente i diritti umani vanno protetti, ma non possono non essere giustificati anche perché non è poi semplice separare attuazione da giustificazione. Senza giustificazione viene a mancare non un motivo qualsiasi, ma un motivo fondamentale proprio per la tutela e la protezione dei diritti umani; il cui futuro è affidato tra l'altro ad una progressiva presa di coscienza delle ragioni che li giustificano. Se i valori ultimi non si giustificano, ma si assumono è segno che tutti i valori si equivalgono e che non c'è un criterio per provare la superiorità o la preferenza di uno rispetto ad altri.

È difficile vedere come ci si possa battere per dei diritti che vengono considerati solo come assunzioni delle quali non è possibile rendere ragione avendo un fondamento contingente, convenzionale, e quindi necessariamente caratterizzato nel senso dell'arbitrarietà, sia esso collettivo (la volontà politica), sia esso individuale (la volontà soggettiva). Allora affermare che l'importante non è fondare i diritti dell'uomo, ma difenderli, significa voler costruirli sulla sabbia. Posizione questa certamente debole, perché i diritti dell'uomo vanno fondati, non per una vaga esigenza culturale o emozionale, ma perché è una esigenza della ragione. $E$ il fondamento dei diritti dell'uomo è innanzitutto e soprattutto di natura etica, la cui dimensione non è dunque una sovrastruttura, essa è parte integrante della persona, è apertura fondamentale all'alterità e al mistero che presiede ogni relazione ed incontro tra gli esseri umani. Né la coscienza può essere considerata, da sola, il luogo di determinazione della legge morale: essa necessita di uno specifico approccio scientifico e filosofico, che illumini certi aspetti della realtà e della condizione umana.

La questione fondamentale e decisiva della fondazione dei diritti umani indica l'esigenza di allargare gli spazi della nostra razionalità, di riaprirla alle grandi questioni del vero, del bene, del giusto, dell'etica della vita, del rispetto della vita, della dignità della persona e quindi di coniugare tra loro la teologia, la filosofia e le scienze, nel pieno rispetto dei loro metodi propri e della loro reciproca autonomia, ma anche della consapevolezza dell'intrinseca unità che li tiene insieme. La considerazione dell'umanità come soggetto naturale ed essenziale e per questo trascenden-

N. Bobbio, II problema della guerra e le vie della pace, Bologna 1979, p. 29.

11 Ibidem, p. 127. Nel capitolo quinto della stessa opera, intitolato Presente e avvenire dei diritti dell'uomo, Bobbio afferma: «ll problema che ci sta dinnanzi, infatti, non è filosofico ma giuridico, è in più largo senso politico» (ibidem, p. 131). Ed ancora: «La dichiarazione universale dei diritti dell'uomo rappresenta la manifestazione dell'unica prova con cui un sistema di valori può essere riconosciuto, e questa prova è il consenso generale circa la sua validità. I giusnaturalismi avrebbero parlato di 'consensus omnium gentium' o 'humani generis' (...). Si stratta di un fondamento, quello storico del consenso che può essere fatalmente provato. Ebbene, la Dichiarazione dei diritti dell'uomo può essere accolta come la più grande prova storica che mai sia stata data, del 'consensus omnium gentium' circa un determinato sistema di valori» (ibidem, pp. 133-134). Cfr. su tale argomento anche F. Di Blasi, Dio e la legge naturale. Una rilettura di Tommaso d'Aquino, Pisa 1999, pp. 32-38. 
tale, fondante i diritti umani, dà, alla stessa architettonica giuridica, le forme della totalità, dell'unità e dell'universalità, non solo per quanto riguarda l'estensione degli stessi diritti, ma anche per la loro applicabilità.

Il formalismo giuridico ha, quale assunto di partenza, la neutralizzazione dell'etica nell'ambito del diritto, ma una teoria del diritto non riguarda solo ciò che il diritto è in se stesso al fine di distinguerlo dal non diritto, ma riguarda anche un ambito che si inscrive all'interno di una filosofia pratica, scuola e politica, sia come presupposto che come conseguenza. ${ }^{12}$

Non si possono salvaguardare i diritti umani, la stessa dignità della persona, prescindendo dalle ragioni profonde in cui essi trovano la loro ultima spiegazione e fondazione, ragione e giustificazione. Queste ultime non possono essere che etico-filosofiche e religiose, perchè fondate sulla struttura ontologica dell'uomo. L'ostacolo reale e di fondo è l'innata resistenza degli Stati a riconoscere che la loro sovranità è e deve essere limitata non già da questa o quella Superpotenza, bensì da un diritto superiore naturale e divino. I diritti dell'uomo perdono cosi il loro preciso fondamento con la negazione del diritto naturale, che di per sè implica il riconoscimento della universalità della natura umana.

Restando abbandonati perciò alla contingenza storica e, in'ultima analisi, alla volontà del legislatore storico, rimangono lettera morta e tutto rimane sulla carta. Una cosa, infatti, sono le Dichiarazioni e le Convenzioni; ed altra cosa è la loro realizzazione pratica, concreta, operativa, se la giustizia internazionale non riuscirà a imporsi e a sovrapporsi alle giurisdizioni nazionali. Quindi se si evita o si rimuove la questione del loro fondamento quelle dichiarazioni finiscono per risultare degli assiomi, sia pure di nobile ordine morale, per i quali è stato possibile solo un accordo pratico, mentre è stato impossibile un accordo teorico. Maritain infatti ricorda

Cfr. F. Viola - G. Zaccaria, Diritto e interpretazioni, Roma-Bari 2002, p. 3. II positivismo giuridico ha pervaso il pensiero giuridico della convinzione che etica e diritto sono su due piani differenti e che il dirito s'identifica con le leggi dichiarate dallo Stato, rispetto alle quali l'etica può influire soltanto nel momento pre-giuridico. La crisi del positivismo rimette in discussione questo assioma e Capograssi introduce la concezione del dirito come esperienza giuridica e quindi non tanto o non come diritto ufficiale statuale, ma come regolamentazione dei rapporti interpersonali sui quali l'etica ha un peso notevole e talora dà ragione e contenuto alla regola politica. II giurista Paolo Grossi afferma che il diritto in quanto ha a che fare con la vita quotidiana dell'uomo non può non avere implicazioni etiche. In questo quadro il "giuridico" appartiene sempre più al diritto e meno alla legge ed è l'etica a chiarire il contenuto della norma che tende a tornare ad essere espressione del volere dei singoli e delle collettività. Grossi afferma: «il diritto non è soltanto ordinamento, ma ordinamento osservato (...), si fonda su una precisa consapevolezza del valore che lo sorregge (...). L'ordine giuridico autentico attinge allo strato dei valori di una comunità per trarne quella forza vitale che nasce unicamente da una convinzione sentita, per trarne quella solidità che non ha bisogno della coazione poliziesca per mantenersi stabile» (P.Grossi, Prima lezione di diritto, Roma-Bari 2003, p. 20). Mentre Kelsen, sebbene usi spesso l'espressione "etico-giuridico", afferma che il diritto non ha nulla di etico e non ha rapporto all'etica: in esso non è ammissibile neanche un "minimo etico» in quanto questo non è un valore essenziale al diritto. La via seguita da Kelsen è quella della neutralità assiologica sia del diritto che della scienza del diritto. Cfr. su tale argomento $H$. Kelsen, La dottrina pura del diritto, tr.it.di M.G. Losano, Torino 1966. 
che l'accordo ci può essere nella formulazione dei diritti, a condizione che non ci si domandi il perché. ${ }^{13}$

Se si nega il sapere metafisico, come il concetto di persona, non è possibile dare una solida fondazione dei diritti dell'uomo che non avrebbero un fondamento oggettivo e solido e si ridurrebbero ad enunciazioni di buona volontà. Per una fondazione stabile e sicura dei diritti dell'uomo c'è bisogno di una spiegazione razionale ed etica che li sottragga alla variabilità e che può trovarsi nella legge naturale insita nella persona umana, creata ad immagine e somiglianza di Dio, proprio perché, per dirla con Rosmini, la persona ha nella sua natura tutti i costitutivi del diritto, in quanto essa è il «diritto sussistente», «l'essenza stessa del diritto» ${ }^{14}$.

13 Cfr. J. Maritain, Introduzione a AA.W., Dei diritti dell'uomo, testi raccolti dell' UNESCO, tr. it. Milano 1952, pp. 1218. Cfr. anche su tale argomento $M$. Indellicato, La centralità della persona in Jacques Maritain, op. cit., in particolare capitoli I e II.

14 Cfr. A.Rosmini, Filosofia del diritto, a cura di R. Orecchia, Padova 1967, pp. 191-192. Giuseppe Capograssi a tal proposito, ha messo in evidenza, con illuminanti osservazioni, il senso unico di questa identificazione della persona umana con il diritto sussistente, e così scrive: "Che significa questa singolare identificazione del diritto con la persona, che a noi abituati come siamo a vedere nel diritto qualche cosa di normativo, di sociale, di collettivo, di coattivo, sembra più che paradossale addirittura incomprensibile? Tutto il concetto di Rosmini, la novità del suo concetto, sta in questa identificazione. E significa: che tutta l'esperienza concreta e storica del diritto, le autorità, le leggi, le obbedienze che la compongono, mettono capo a questo unico punto vivo che è la persona (...). Questa specie di anello magico dell'infinito e dell'empirico, è, come tale, attività e sovranità: attività perché è la vita stessa che si muove e si organizza in tutte le sue concrete esigenze e sovranità perché è l'affermazione della vita come verità cioè della sua destinazione infinita. Per conseguenza la persona è il diritto sussistente, perché nella persona verità e vita, eterno e sentimento, valore e fatto fanno blocco: per essa realizzare la propria vita è adempire un infinito dovere nel quale si riassumono tutti i doveri della vita. E quindi in essa, per usare il linguaggio preciso dei giuristi, diritto oggettivo e diritto soggettivo coincidono: l'affermazione della propria vita come tendenza alla felicità e l'affermazione della vita come legge assoluta (...). I fatti fondamentali e costitutivila esistenza della persona, e la congiunzione che la persona fa a se stessa, al suo principio supremo, delle sue attività particolari che così diventano proprie, e attraverso tali attività la congiunzione che essa fa a se stessa alla qualche la sua sete dell'essere la spinge, delle cose, delle volontà, delle persone- costituiscono l'essenza stessa e il sistema dei diritti. II diritto è la persona. Egli ci ha detto una verità la qualche secondo la natura della verità è ricca di innumerevoli contenuti ed inesauribile nella sua ricchezza. E' una verità che Rosmini ci ha detto, ma è una verità che se non tutti riconoscono, tutti effettivamente seguono e la storia del pensiero giuridico lo dimostra»" (G. Capograssi, II diritto secondo Rosmini, in Opere, Milano 1959, vol. IV, pp. $331 \mathrm{ss).} \mathrm{Questa} \mathrm{lapidaria} \mathrm{formula}$ della persona "diritto sussistente" non rappresenta una definizione retorica o vaga ma è una espressione densa di significato: il diritto è antropologicamente ed assiologicamente fondato, e la persona-diritto esprime il generale principio ontologico di sussistenza. II diritto è pensabile come un modo d'essere dell'uomo, diciamo pure che non esistendo fuori dell'uomo e senza dell'uomo, il diritto è un atto "ontologico"nel quale l'uomo progetta e realizza la condizione della convivenza con un minimo di interiorità e un massimo di esteriorità relazionale. "Nel quadro del principio ontologico di sussistenza, - afferma - Sergio Cotta, Rosmini individua la "supremazia naturale" della persona nell'ordine naturale e sociale poiché sul piano conoscitivo essa ha "la potenza di affermare tutto l'essere". Ne consegue, sul piano direttivo- retto dal principio di "riconoscere (nell'agire) le cose per quelle che sono" - il dovere unitario (morale, giuridico, politico) di rispettare la persona nella sua sussistenza. In questi termini, la costruzione rosminiana risulta perfettamente coerente e rivelatrice del fondamento dei diritti dell'uomo. La persona, l'individuo umano, è la realtà ontologica prima, il principio ( non nel senso di assioma bensì di arké) nell'ordine tanto dell'umana riflessione teoretica e coscienziale, quanto dell' agire pratico in ogni sua forma» (S. Cotta, La persona come "diritto sussistente", oggi, in AA. W., Rosmini pensatore europeo, Atti del Congresso internazionale, Roma 26-29 ottobre 1988, Milano 1989, p. 177). Per un'etica della vita esigente il diritto, è al diritto naturale che bisogna far riferimento innanzitutto, come all'apriori ontoassiologico-universale antropologi$\mathrm{co}$, perché «il diritto, come afferma Aldo Moro, è essenzialmente la stessa legge etica come verità e perciò determinante di un completo processo di attuazione della totale vita etica della umanità» (A. Moro, Lo Stato. II Diritto. Bari 2006, p. 45). II mondo umano è anche essenzialmente giuridico a titolo della sua propria umanità, in ragione cioè di quella vibrazione etica che lo pervade e ne costituisce l'anima di libertà. "Se ci sono diritti soggettivi, non è solo perché prima ci sono norme giuridiche che li istituiscono e li garantiscono con l'usbergo della sanzione minacciata a chi osa violarli (...). La linfa dei diritti soggettivi, e non esistono diritti più soggettivi dei diritti dell'uomo, precede le norme giuridiche dei codici e delle leggi. Ed erompe, i codici e le leggi non essendo che crosta di un 
Alla base dei valori essenziali vi è solo il riconoscimento di una legge morale che, in quanto "legge naturale" iscritta nel cuore dell'uomo, per dirla con Maritain, è punto di riferimento normativo della stessa legge civile. Quando, per un tragico oscuramento della coscienza collettiva, lo scetticismo giungesse a porre in dubbio persino i principi fondamentali della legge, lo stesso ordinamento democratico sarebbe scosso e minato nelle sue fondamenta. Scrive Giovanni Paolo II: « $\mathrm{Al}$ di là delle intenzioni, talvolta buone, e delle circostanze, spesso difficili, le autorità civili e i soggetti particolari non sono mai autorizzati a trasgredire i diritti fondamentali e inalienabili della persona umana. Così solo una morale che riconosce delle norme valide sempre e per tutti senza alcuna eccezione, può garantire il fondamento etico della convivenza sociale sia nazionale che internazionale ${ }^{15}$. 


\section{IL FONDAMENTO ETICO DEI DIRITTI UMANI}

La questione fondamentale e decisiva della fondazione dei diritti umani indica l'esigenza di allargare gli spazi della nostra razionalità, di riaprirla alle grandi questioni del vero, del bene, del giusto, dell'etica della vita, del rispetto della vita, della dignità della persona e quindi di congiungere tra loro la teologia, la filosofia e le scienze, nel pieno rispetto dei loro metodi propri e della loro reciproca autonomia, ma anche della consapevolezza dell'intrinseca unità che li tiene insieme.

Non è possibile salvaguardare i diritti umani, la stessa dignità della persona, prescindendo dalle ragioni profonde in cui essi trovano la loro ultima spiegazione e fondazione, ragione e giustificazione. Queste ultime non possono essere che etico-religiose perché fondate sulla struttura ontologica dell'uomo. Il diritto è la persona e la persona è la realtà onto-assiologica prima, il principio nell'ordine tanto dell'umana riflessione teoretica e coscienziale, quanto dell'agire pratico in ogni sua forma. 


\section{ETYCZNE PODSTAWY PRAW CZŁOWIEKA}

Fundamentalną oraz decydującą kwestią podczas ustanawiania praw człowieka jest potrzeba rozszerzenia zakresu naszej racjonalności i ponownego otworzenia jej na ważne kwestie prawdy, dobra, sprawiedliwości, etyki życia, godności ludzkiej, a więc połączenia ze sobą teologii, filozofii i nauki przy pełnym poszanowaniu ich własnych metod i ich wzajemnej autonomii, jak i również przy świadomości ich wewnętrznej jedności, która utrzymuje je razem.

Nie ma możliwości ochrony praw człowieka, samej godności osoby, nie biorąc pod uwagę głębokich przyczyn, w których znajdują one ostateczne wyjaśnienie oraz swoje ustanowienie, powód i wytłumaczenie. Te ostatnie mają charakter etycznoreligijny ponieważ opierają się na strukturze ontologicznej człowieka. Prawo jest osobą a osoba jest najpierw rzeczywistością onto-aksjologiczną zasadą w porządku stworzoną na podstawie zarówno refleksji teoretycznej i sumienia ludzkiego, jak i praktycznego działania w każdej formie. 


\section{THE ETHICAL BASIS OF HUMAN RIGHTS}

The fundamental and decisive factor in establishing human rights, is the need to extend the scope of our rationality and to re-open it on important issues of truth, good, justice, ethics of life, human dignity, and therefore the fusion of theology, philosophy and science with full respect for their own methods and their mutual autonomy, and also with the awareness of their inner unity, which keeps them together.

There is no possibility of protection of human rights, the dignity of a person, not taking into account the profound causes, in which they have their definitive explanation and establishment, the reason and explanation. The latter are of ethical and religious character because they are based on the ontological structure of the human being. The right is the person and the person is the onto-axiological reality at first, the principle of the order created on the base of both theoretical reflection and human conscience, and practical action in any form.

Key words: human rights, theology, philosophy, science, rationality 